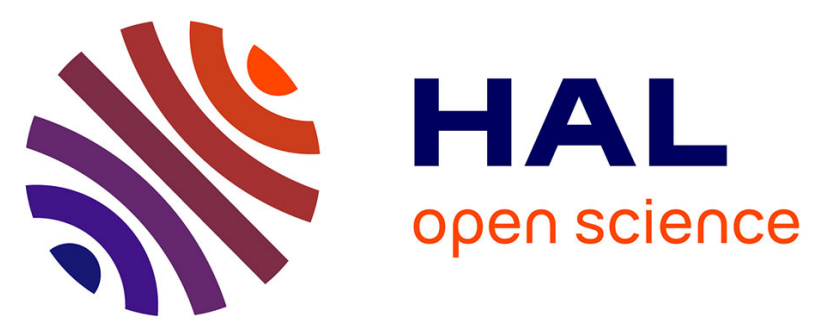

\title{
An optimized alkyl chain-based binding motif for 2D self-assembly: a comprehensive crystallographic approach
}

David Bléger, Amandine Bocheux, David Kreher, Fabrice Mathevet, André-Jean Attias, Germain Metgé, Ludovic Douillard, Céline

Fiorini-Debuisschert, Fabrice Charra

\section{To cite this version:}

David Bléger, Amandine Bocheux, David Kreher, Fabrice Mathevet, André-Jean Attias, et al.. An optimized alkyl chain-based binding motif for 2D self-assembly: a comprehensive crystallographic approach. Nanoscale, 2013, 5, pp.1452 - 1455. 10.1039/c2nr33509c . cea-01478816

\section{HAL Id: cea-01478816 https://hal-cea.archives-ouvertes.fr/cea-01478816}

Submitted on 28 Feb 2017

HAL is a multi-disciplinary open access archive for the deposit and dissemination of scientific research documents, whether they are published or not. The documents may come from teaching and research institutions in France or abroad, or from public or private research centers.
L'archive ouverte pluridisciplinaire HAL, est destinée au dépôt et à la diffusion de documents scientifiques de niveau recherche, publiés ou non, émanant des établissements d'enseignement et de recherche français ou étrangers, des laboratoires publics ou privés. 
Cite this: Nanoscale, 2013, 5, 1452

Received 6th November 2012

Accepted 28th December 2012

DOI: $10.1039 / c 2 n r 33509 c$

\title{
An optimized alkyl chain-based binding motif for 2D self-assembly: a comprehensive crystallographic approach $\dagger$
}

\author{
David Bléger, ${ }^{\star \mathrm{a}}$ Amandine Bocheux, ${ }^{\mathrm{b}}$ David Kreher, ${ }^{\mathrm{a}}$ Fabrice Mathevet, ${ }^{\mathrm{a}}$ \\ André-Jean Attias, ${ }^{\text {*a }}$ Germain Metgé, ${ }^{\mathrm{b}}$ Ludovic Douillard, ${ }^{\mathrm{b}}$ Céline Fiorini- \\ Debuisschert $^{\mathrm{b}}$ and Fabrice Charra ${ }^{* \mathrm{~b}}$
}

www.rsc.org/nanoscale

\begin{abstract}
Taking into account substrate crystallographic constraints, an overarching molecular binding motif has been designed to allow transferable self-assembling patterns on different substrates. This optimized clip demonstrates robust and equivalent self-assembled architectures on both highly oriented pyrolitic graphite (HOPG) and reconstructed $\mathrm{Au}(111)$ surfaces.
\end{abstract}

The realization of highly organized two-dimensional (2D) selfassembled monolayers physisorbed on atomically flat (semi) conducting surfaces ${ }^{\mathbf{1 - 4}}$ is an important objective in nanotechnology. Such surface-confined architectures are commonly engineered by using supramolecular interactions, i.e. metalligand, electrostatic ionic, hydrogen bonding, or van der Waals forces. Building blocks equipped with carefully designed binding motifs based on these interactions can lead to preprogrammed patterns. Despite its many advantages (ease of preparation, high order of the monolayers, feasibility of complex patterns ${ }^{5}$ ), some limitations of the 2D self-assembly bottom-up approach still remain. For example, preparing architectures which are robust under ambient conditions is rather difficult and for this purpose combining physisorption with chemisorption strategies has proven to be beneficial. ${ }^{6}$ Additionally, most of the binding motifs are designed for a specific substrate and difficult to transfer to another one. This latter limitation is especially impeding when a particular function is targeted and requires specific substrates. Such is the case for molecular plasmonic applications which require noble metal substrates like $\mathrm{Au}(111)$.

\footnotetext{
${ }^{a}$ Laboratoire de Chimie des Polymères, Université Pierre et Marie Curie, UMR 7610, 3 rue Galilé, F-94200 Ivry, France.E-mail: andre-jean.attias@ upmc.fr

${ }^{b}$ Service de Physique et Chimie des Surfaces et Interfaces, CEA, IRAMIS, F-91191 Gif sur Yvette, France. E-mail: fabrice.charra@cea.fr

$\dagger$ Electronic supplementary information (ESI) available: Synthesis and experimental details. See DOI: $10.1039 / \mathrm{c} 2 \mathrm{nr} 33509 \mathrm{c}$

$\ddagger$ Present address: Department of Chemistry, Humboldt-Universität zu Berlin, Brook-Taylor-Str. 2, 12489 Berlin, Germany, E-mail: david.bleger@ chemie.hu-berlin.de
}

Though rather weak (typically $0.02-0.1 \mathrm{eV}$ ) compared to some other non-covalent interactions, van der Waals forces nevertheless play a crucial role in the formation of $3 \mathrm{D}$ as well as 2D supramolecular systems. At surfaces they promote the physisorption of organic species and can lead to strong lateral interactions between molecules. A typical example is linear hydrocarbons which form stable monolayers on highly oriented pyrolitic graphite (HOPG) under ambient conditions, i.e. at the interface with air or liquids. According to the Groszek model, ${ }^{7}$ $n$-alkanes are oriented along the $\langle 100\rangle$ direction of graphite leading to close-packed lamellar structures. ${ }^{8}$ The robustness of such monolayers results from two factors. First, the good registry between alkyl chains (the period which includes two methylene $\left(\mathrm{CH}_{2}\right)$ groups is $\left.2.51 \AA\right)$ and the HOPG lattice unit (period of $2.46 \AA$ along $\langle 100\rangle$ ), which leads to a cumulative adsorption energy of about $65 \mathrm{meV}$ per methylene group. ${ }^{9}$ Second, the attractive lateral interaction between adsorbed molecules, which is on the order of $25 \mathrm{meV}$ per pair of facing methylene groups. ${ }^{10}$ As a consequence, the building blocks composed of aromatic cores functionalized with peripheral alkyl chains can usually form stable monolayers on HOPG by interdigitation of their alkyl chains. On $\mathrm{Au}(111) n$-alkanes can also form stable lamellar structures, ${ }^{\mathbf{1 1 - 1 4}}$ although their assemblies are much more sensitive to the number of methylene groups per chain, ${ }^{13}$ an effect explained by the incommensurability of the lattice of linear hydrocarbons with the Au(111) (on reconstructed $\mathrm{Au}(111)$ the distance between neighboring atoms of the topmost atomic layer is $2.75 \AA$ or $2.85 \AA$ depending on their orientation relative to surface-reconstruction). Although some examples have been reported where $\pi$-conjugated moieties such as porphyrins, ${ }^{15}$ triphenylenes, ${ }^{16,17}$ or dehydrobenzo [12]annulene ${ }^{18}$ equipped with lateral alkyl chains can form wellordered self-assembled monolayers on both HOPG and Au(111), the crystallographic differences between those substrates make the development of an overarching binding motif, able to steer 2D self-assemblies on various substrates, highly challenging.

Recently we developed a functional "clip" (see Fig. 1a), specifically designed for 2D supramolecular self-assembly 
a) Non-optimized "clip »

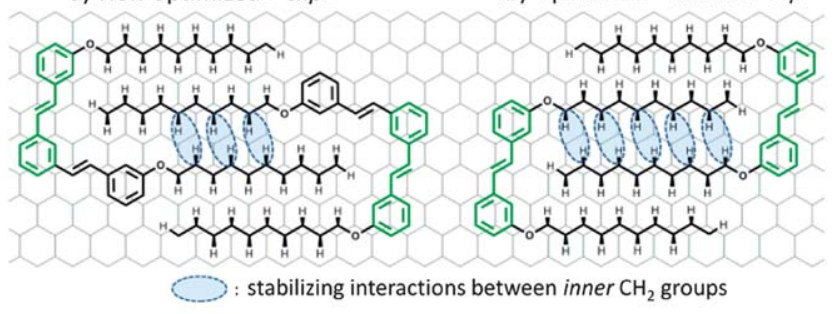

c)

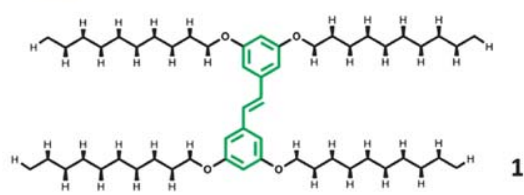

Fig. 1 "Minimal clip" design and principle. (a) The non-optimized clip"19-21 and (b) the minimal clip optimized for packing interactions between interdigitated alkyls on HOPG. (c) Chemical structure of the smallest bifunctional unit (1).

confined on HOPG. ${ }^{19}$ This recognition motif consists of two alkyl chains held at a distance close to $8.52 \AA$ (i.e. twice the lateral distance between $n$-alkanes physisorbed on HOPG) from each other by a rigid linker. The clip was designed in such a way that when two of these units interdigit on the surface, their selfassembly leads to the formation of lamellae that strictly preserve the Groszek geometry. The formation of such lamellae is the driving force of the self-assembly processes. This binding motif is able to steer the self-assembly of a large variety of tectons resulting in the formation of $2 \mathrm{D}$ supramolecular architectures of different topologies. ${ }^{19-21}$ However, the clipping functional group suffers from two drawbacks: (i) the rotations around the $\sigma$-bonds of the two stilbene units lead to various conformations, which is detrimental from the entropic point of view and (ii) the lateral shift between inner decyloxy chains only three $\mathrm{CH}_{2}$ out of five potential ones are involved in the clipping process (see Fig. 1a) - which induces a non-optimized clipping energy.

Here we revisit our clip concept and present a new binding motif optimized for adsorption on HOPG yet capable of directing the formation of self-assemblies on $\mathrm{Au}(111)$ at the solid-liquid interface. This so-called "minimal clip" (see Fig. 1b) comprises, as a linker between the two alkyl chains, a new $\pi$-conjugated bridge rigidified through the use of a single stilbene unit instead of two in the non-optimized version. Within this new design, the minimal clip presents less rotational degrees of freedom which is favorable to the formation of more stable ordered phases. Most importantly, the lateral shift between inner decyloxy chains is suppressed, with all the internal methylenes involved in the clipping process thus enhancing the clipping energy, a crucial feature for adsorption on $\mathrm{Au}(111)$. Moreover, the same reduced conjugated skeleton is able to bear two parallel clips, hence optimizing the compactness and the surface density of clipped motifs. As a first example, the smallest bifunctional building block equipped with the minimal clip (1, see Fig. 1c) has been synthesized and successfully self-assembled on HOPG and $\mathrm{Au}(111)$ as demonstrated with scanning tunneling microscopy (STM).
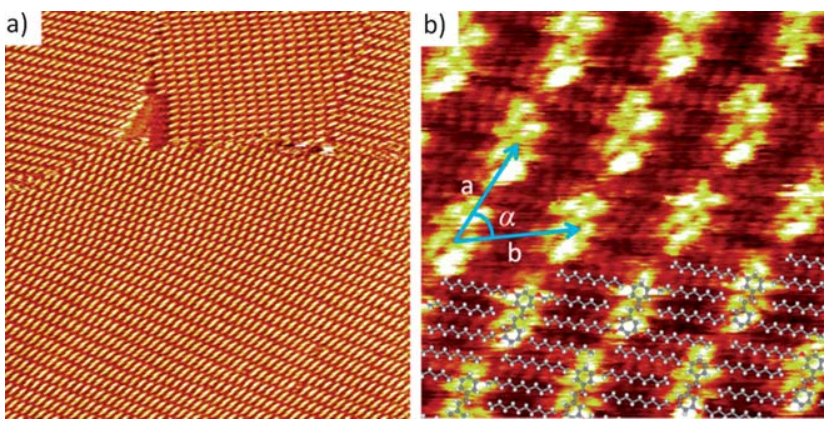

Fig. 2 Self-assembly of $\mathbf{1}$ into non-covalent polymer-like structures at the HOPG-phenyloctane interface at room temperature. (a) Larger-scale $(74.4 \times 74.4$ $\left.\mathrm{nm}^{2}\right)$ and (b) high-resolution $\left(6.5 \times 6.5 \mathrm{~nm}^{2}\right)$ STM images; a scheme of the packing model is superimposed, together with the definition of cell parameters $\left(a=1.86 \mathrm{~nm}, b=2.01 \mathrm{~nm}, \alpha=54^{\circ}\right.$ ). Imaging parameters: (a) $V_{\text {bias }}=-1.2 \mathrm{~V}$ and $I_{\mathrm{t}}=61 \mathrm{pA}$, (b) $V_{\text {bias }}=-1.2 \mathrm{~V}$ and $I_{\mathrm{t}}=93 \mathrm{pA}$.

On HOPG highly reproducible monolayers with large domains were observed after deposition of $\mathbf{1}$ from phenyloctane at room temperature (see Fig. 2a). The bright features are attributed to the aromatic cores and form a periodic pattern on the substrate. Intramolecular resolution (see Fig. $2 \mathrm{~b}$ ) was obtained by scanning a smaller area in the height mode (constant current). Linear features appear in the dark regions and may be attributed to the aliphatic chains. As anticipated for a bifunctional molecule, i.e. a building block bearing two binding motifs, 1D supramolecular chains composed of clipped molecules are formed (see the superimposed model). The chains are themselves closely packed and interact in an optimized geometry in order to maximize their density on the surface. The distance between the nearest neighbors within the same row is $b_{\mathrm{HOPG}}=2.01 \pm 0.08 \mathrm{~nm}$, which is in good agreement with the value inferred from the theoretical model represented in Fig. $1 \mathrm{~b}\left(b_{\text {theo }}=2.04 \mathrm{~nm}\right)$. Therefore, all the alkyl chains are physisorbed in a linear and flat fashion on the surface of HOPG, and most importantly, all the inner $\mathrm{CH}_{2}$ are now facing each other leading to a maximized interdigitation of the alkyl chains, and thus to an improved structure stabilization through their optimized lateral interaction.

On $\mathrm{Au}(111)$ a similar self-assembly behavior was observed. Monodomains of $\mathbf{1}$ are formed over relatively large areas (see Fig. 3a). Simultaneous imaging of the monolayer and of the $22 \times \sqrt{3} \mathrm{Au}(111)$ surface reconstruction (a $4.2 \%$ shrink of the topmost atomic layer compared to the underlying bulk crystallographic period along the $\langle 110\rangle$ direction) allows for determining the orientation of the molecules with respect to the substrate lattices. This is also exploited to confirm - or improve - the calibration of length measurements, the lateral period between chevrons being taken as $22 a=6.34 \mathrm{~nm}$, with $a$ being the interatomic distance in the bulk. Like with HOPG, the alkyl chains on $\mathrm{Au}(111)$ adopt the same orientation as adsorbed $n$-alkanes do, ${ }^{12}$ i.e. along one of the $\langle 110\rangle$ directions of $\mathrm{Au}(111)$. Higher resolution images (see Fig. $3 \mathrm{~b}$ ) show that the molecules are clipped with each other forming rectilinear supramolecular chains similar to the ones observed on HOPG. However, beside 
this qualitative similarity, significant quantitative changes are observed in the case of $\mathrm{Au}(111)$. First, the distance between clipped molecules $\left(b_{\mathrm{Au}(111)}=1.86 \pm 0.03 \mathrm{~nm}\right)$ is significantly smaller than the one measured on HOPG $\left(b_{\mathrm{HOPG}}=2.01 \mathrm{~nm}\right)$ whereas the distance between clipped rows is increased from $a$ $\sin \alpha=1.50 \mathrm{~nm}$ (HOPG) to $1.77 \mathrm{~nm}(\mathrm{Au})$ leading to a loss of coverage from 0.33 molecule $\mathrm{nm}^{-2}$ (HOPG) to 0.30 molecule $\mathrm{nm}^{-2}(\mathrm{Au})$. A closer inspection of the images reveals that, in contrast to HOPG on which the alkyl chains are adsorbed in a straight conformation, the chains on $\mathrm{Au}(111)$ are distorted (see Fig. $3 \mathrm{~b}$ and $\mathrm{c}$ ). All these points can be explained based on crystallographic grounds. As a matter of fact, the measured period between alkyl chains, taken in the perpendicular crystallographic direction, increases from $0.426 \pm 0.016 \mathrm{~nm}$ on HOPG to $0.500 \pm 0.02 \mathrm{~nm}$ on $\mathrm{Au}(111)$, a difference much beyond measurement uncertainties. The latter is consistent with the crystallographic distance between two equivalent $\langle 110\rangle$ rows, which amounts to $0.482 \mathrm{~nm}$, accounting for the orientation of surface reconstruction present in Fig. 3a. Moreover, just like on HOPG (Groszek model), linear alkanes are known to pack parallel to each other, adsorbed in a commensurate order on $\mathrm{Au}(111)$ that is on equivalent $\langle 110\rangle$ substrate rows. ${ }^{13}$ Owing to the inherent flexibility of the alkyl chain and the oxygen link it is thus legitimate to interpret the structure adopted by the alkyl chain as adsorption mimicking linear alkanes, as observed on HOPG. However, in the case of $\mathrm{Au}(111)$, they have to move apart from one another so as to reach the next adsorption $\langle 110\rangle$ row thus preserving their registry. The conjugated backbone onto which they are linked is more rigid, as confirmed by the very similar apparent elongations of their STM images on HOPG and $\mathrm{Au}(111)$, both about $1.2 \mathrm{~nm}$, a value consistent with the end-toend distance between the $\mathrm{H}$-termini in the gas phase $(1.1 \mathrm{~nm})$. Hence, the alkyl chains, including their alkoxy linker, have to bend in order to reach the nearest $\langle 110\rangle$ row which allows the most stable adsorption. This is clearly evidenced in a highresolution image (Fig. 3b) where the locations of two neighboring rows are indicated by blue dotted lines and where the bending of the alkyl chains is visible in the STM image. The epitaxy of the alkyl chains forces all molecules to be adsorbed in equivalent sites, which is supported by the fact that all the molecules have the same contrast on the STM images, whereas the images of such planar aromatic molecules are known to be strongly site-dependent.

A deeper analysis of the monolayers obtained on $\mathrm{Au}(111)$ shows that although the structure of the supramolecular chains of clipped molecules (in particular the lattice vector $\boldsymbol{b}$ ) is strictly constant, their packing geometry, and thus the lattice vector $\boldsymbol{a}$, is subject to variations from place to place. This is highlighted in Fig. 3b which has been selected to show different packing variants. Those variants are obtained through a translation along the alkyl chain adsorption rows, from end-to-end fullyadsorbed alkyls chains (the blue circle in Fig. $3 \mathrm{~b}$ ) up to fully superimposed chains sharing the same adsorption site (the white circle in Fig. 3b). In the latter case, the alkyl chains share a common adsorption row which imposes a partial adsorption. Such partial adsorptions of alkyl chains and the corresponding increased density were already reported in other comparative
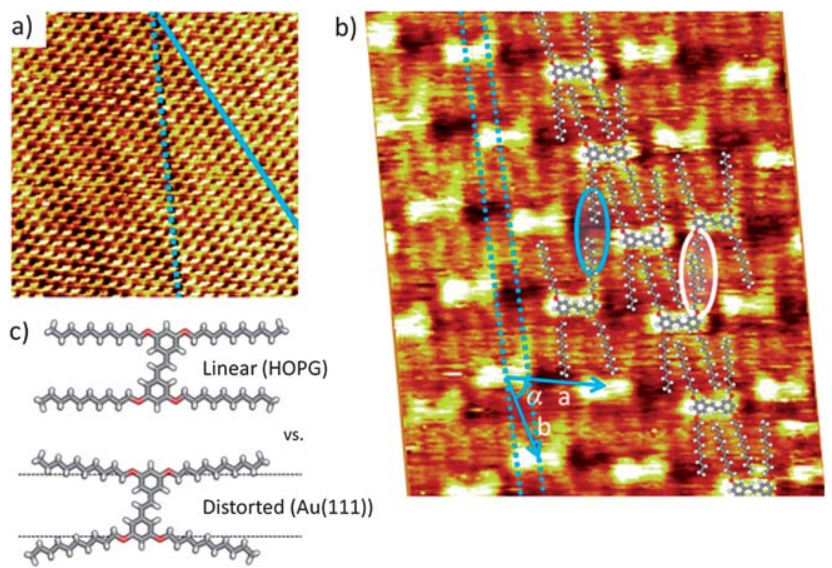

Fig. 3 Self-assembly of $\mathbf{1}$ into non-covalent polymer-like structures at the $\mathrm{Au}(111)$-phenyloctane interface at room temperature. (a) Large-scale (34.0 $\times$ $\left.34.0 \mathrm{~nm}^{2}\right)$ STM image $\left(V_{\text {bias }}=-1.00 \mathrm{~V}\right.$ and $\left.I_{\mathrm{t}}=25 \mathrm{pA}\right)$; the $\langle 110\rangle$ (blue dotted line) and $\langle 112\rangle$ (blue solid line) directions are represented, the latter is parallel to the trace of gold reconstruction lines. (b) High-resolution $\left(8.5 \times 10.0 \mathrm{~nm}^{2}\right)$ STM image $\left(V_{\text {bias }}=-1.10 \mathrm{~V}\right.$ and $\left.l_{\mathrm{t}}=25 \mathrm{pA}\right)$; the frame of the image is slanted as a result of a software drift-correction; a scheme of cell parameters is superimposed ( $a=2.05$ $\left.\mathrm{nm}, b=1.86 \mathrm{~nm}, \alpha=60^{\circ}\right)$; two equivalent lines parallel to the $\langle 110\rangle$ direction of $\mathrm{Au}(111)$ are represented by blue dotted lines; their distance is $0.5 \mathrm{~nm}$ and they form an angle of $19^{\circ}$ with respect to the vector $\boldsymbol{b}$; two different packing variants of the noncovalent polymer chains are highlighted in blue and white. (c) Schematic representation of $\mathbf{1}$ adsorbed with either linear (as observed on HOPG) or distorted (as observed on $\mathrm{Au}(111)$ reconstruction) alkyl chains.

studies with HOPG and $\mathrm{Au}(111)$ as substrates. ${ }^{15-17}$ It is remarkable that the two main driving forces of the self-assembly, i.e. molecule-substrate interactions (adsorption of the alkyl chains following an epitaxial relationship with the $\mathrm{Au}(111))$ and molecule-molecule interactions (formation of the clip), work in concert to form rectilinear chains of clipped molecules constituting the most stable entity of the molecular assembly.

Moreover, gold surface reconstruction plays a prominent role in alkyl chain orientation. Inspection of several images shows that alkyl chains are almost never aligned along the specific $\langle 110\rangle$ direction which is parallel to direction of the shrink associated with the $\mathrm{Au}(111)$ surface reconstruction. This observation is consistent with organization of linear alkanes on the reconstructed $\mathrm{Au}(111)$. Indeed, among the three possible $\langle 110\rangle$ adsorption rows of $\mathrm{Au}(111)$, the one aligned along the shrinked direction of the $(22 \times \sqrt{3})$ surface reconstruction presents a finite corrugation and is thus unfavorable to accommodate a linear molecule. ${ }^{12}$ Moreover, in the present case, this trend is even more pronounced because alkyl chains are linked to a rigid core. Actually, whereas for alkyl chains oriented along the shrink direction the distance between neighbouring alkyl chain adsorption sites is unchanged compared to the bulk, that is $0.498 \mathrm{~nm}$, for the other two directions $\left( \pm 60^{\circ}\right.$ from the shrink direction) it is reduced to $0.482 \mathrm{~nm}$. The latter being significantly closer to the distance between their linking points on the backbone, about $0.43 \mathrm{~nm}$, the alkyl-chain bending and the associated stress energy are reduced for the corresponding domain orientations which are thus favoured. This preferential orientation of the alkyl chain 
axis may explain the lack of organization obtained on $\mathrm{Au}(111)$ with some other molecules based on clips and forming wellorganized networks on HOPG. For instance, the tristilbene building blocks which bear three clips at $120^{\circ}$ from one another ${ }^{19}$ and form chicken-wire alveolar networks on HOPG cannot adsorb on $\mathrm{Au}(111)$ without some of the alkyl chains being adsorbed along this specific reconstruction shrinking $\langle 110\rangle$ direction. Consistently, such networks have never been observed on this substrate. Similarly, as mentioned earlier, alkyl-substituted conjugated-cored tectons with 3-fold symmetry ${ }^{17,18}$ form a hexagonal network on HOPG, whereas a completely different phase is observed on $\mathrm{Au}(111)$ the latter being consistent with the existence of a "forbidden" adsorption direction. Exclusion of systems requiring alkyl chain adsorption along all three $\langle 110\rangle$ directions thus constitutes another guideline for the design of clip-based architectures destined to reconstructed $\mathrm{Au}(111)$ substrates.

Finally, the robustness of the monolayers formed by 1 on both substrates was investigated through washing experiments carried out in different solvents. On HOPG the supramolecular polymer-like structures formed by 1 could be washed several times with phenyloctane without affecting their self-assembly, i.e. the same patterns were observed with STM before and after rinsing the substrate with this solvent (see Fig. S2a†). Such a high resistance to multiple washings demonstrates the robustness of the self-assembled structures formed by $\mathbf{1}$ on graphite. On the other hand, monolayers formed by $\mathbf{1}$ on $\mathrm{Au}(111)$ are moderately resistant to washings with phenyloctane. After rinsing the surface twice with this solvent, some well-organized domains remain but most of the molecules have been desorbed (see Fig. S2b $\dagger$ ). Washing the substrates with a better solvent such as THF results in the total desorption of the monolayers on both substrates. Hence, even if the minimal clip is able to drive the self-assembly of 1 on $\mathrm{Au}(111)$, its adsorption energy remains weaker on this substrate than on HOPG.

In summary, we have designed a new surface-confined binding motif - "minimal clip" - by maximizing the interdigitation of linear alkyl chains on atomically planar surfaces. The use of this optimized clip allowed us to steer self-assemblies of the same molecular building block on two different substrates, i.e. HOPG and $\mathrm{Au}(111)$. Bifunctional unit 1 forms large monodomains consisting of closely packed non-covalent polymer-like structures on both surfaces. The details of the clip-forming adsorption geometry on the reconstructed $\mathrm{Au}(111)$ have been determined, which enabled us to derive specific design rules which are adapted to the specific crystallography of this substrate. Thanks to improved 2D adsorption energy and lateral interactions, the minimal clip allows for constructing monolayers with enhanced robustness under ambient conditions, and pattern different surfaces with the same building block, i.e. at a limited cost.

\section{Acknowledgements}

The french "Agence Nationale de la Recherche" (ANR) is acknowledged for its generous support through the NOMAD project of the PNano program.

\section{Notes and references}

1 J. V. Barth, Annu. Rev. Phys. Chem., 2007, 58, 375.

2 S. Furukawa and S. De Feyter, Top. Curr. Chem., 2009, 287, 87.

3 L. Bartels, Nat. Chem., 2010, 2, 87.

4 A. Ciesielski and P. Samori, Nanoscale, 2011, 3, 1397.

5 J. Adisoejoso, K. Tahara, S. Okuhata, S. Lei, Y. Tobe and S. De Feyter, Angew. Chem., Int. Ed., 2009, 48, 7353.

6 R. Madueno, M. T. Raisanen, C. Silien and M. Buck, Nature, 2008, 454, 618.

7 A. J. Groszek, Proc. R. Soc. London, Ser. A, 1970, 314, 473.

8 J. P. Rabe and S. Buchholz, Science, 1991, 253, 424.

9 A. J. Gellman and K. R. Paserba, J. Phys. Chem. B, 2002, 106, 13231.

10 S. X. Yin, C. Wang, X. H. Qiu, B. Xu and C. L. Bai, Surf. Interface Anal., 2001, 32, 248.

11 K. Uosaki and R. Yamada, J. Am. Chem. Soc., 1999, 121, 4090.

12 R. Yamada and K. Uosaki, J. Phys. Chem. B, 2000, 104, 6021.

13 A. Marchenko, J. Cousty and L. P. Van, Langmuir, 2002, 18, 1171.

14 H. M. Zhang, Z. X. Xie, B. W. Mao and X. Xu, Chem.-Eur. J., 2004, 10, 1415.

15 N. Katsonis, J. Vicario, T. Kudernac, J. Visser, M. M. Pollard and B. L. Feringa, J. Am. Chem. Soc., 2006, 128, 15537.

16 N. Katsonis, A. Marchenko and D. Fichou, J. Am. Chem. Soc., 2003, 125, 13682.

17 K. Perronet and F. Charra, Surf. Sci., 2004, 551, 213.

18 T. Balandina, K. Tahara, N. Sändig, M. O. Blunt, J. Adisoejoso, S. Lei, F. Zerbetto, Y. Tobe and S. De Feyter, ACS Nano, 2012, 6, 8381.

19 D. Bléger, D. Kreher, F. Mathevet, A.-J. Attias, G. Schull, L. Douillard, A. Huard, C. Fiorini-Debuisschert and F. Charra, Angew. Chem., Int. Ed., 2007, 46, 7404.

20 D. Bléger, D. Kreher, F. Mathevet, A.-J. Attias, I. Arfaoui, G. Metgé, L. Douillard, C. Fiorini-Debuisschert and F. Charra, Angew. Chem., Int. Ed., 2008, 47, 8412.

21 D. Bléger, F. Mathevet, D. Kreher, A. J. Attias, A. Bocheux, S. Latil, L. Douillard, C. Fiorini-Debuisschert and F. Charra, Angew. Chem., Int. Ed., 2011, 50, 6562. 